\title{
Turbo Demodulation for LDPC-coded High-order QAM in Presence of Transmitter Angular Skew
}

\author{
Koike-Akino, T.; Millar, D.S.; Kojima, K.; Parsons, K.; Yoshida, T.; Ishida, K.; Miyata, Y.; \\ Matsumoto, W.; Mizuochi, T.
}

TR2014-086 September 2014

\begin{abstract}
We analyze demodulation methods for high-order QAM formats in the presence of quadrature angular skew caused by imperfect biasing of the transmitter. Proposed turbo demodulation improves skew tolerance of up to 33-degree angle for an SNR penalty of $1 \mathrm{~dB}$ for 1024QAM.

European Conference on Optical Communications (ECOC)
\end{abstract}

This work may not be copied or reproduced in whole or in part for any commercial purpose. Permission to copy in whole or in part without payment of fee is granted for nonprofit educational and research purposes provided that all such whole or partial copies include the following: a notice that such copying is by permission of Mitsubishi Electric Research Laboratories, Inc.; an acknowledgment of the authors and individual contributions to the work; and all applicable portions of the copyright notice. Copying, reproduction, or republishing for any other purpose shall require a license with payment of fee to Mitsubishi Electric Research Laboratories, Inc. All rights reserved. 



\title{
Turbo Demodulation for LDPC-Coded High-Order QAM in Presence of Transmitter Angular Skew
}

\author{
Toshiaki Koike-Akino(1), David S. Millar(1), Keisuke Kojima ${ }^{(1)}$, Kieran Parsons ${ }^{(1)}$, \\ Tsuyoshi Yoshida ${ }^{(2)}$, Kazuyuki Ishida(3), Yoshikuni Miyata(2), Wataru Matsumoto(2), Takashi Mizuochi(4) \\ (1) Mitsubishi Electric Research Labs., 201 Broadway, Cambridge, MA 02139, USA, koike@merl.com \\ (2) Information Technology R\&D Center, Mitsubishi Electric Corp., 5-1-1, Ofuna, Kamakura, Japan \\ (3) Communication Networks Center, Mitsubishi Electric Corp., 8-1-1, Tsukaguchi, Amagasaki, Japan \\ (4) Advanced Technology R\&D Center, Mitsubishi Electric Corp., 8-1-1, Tsukaguchi, Amagasaki, Japan
}

\begin{abstract}
We analyze demodulation methods for high-order QAM formats in the presence of quadrature angular skew caused by imperfect biasing of the transmitter. Proposed turbo demodulation improves skew tolerance of up to 33-degree angle for an SNR penalty of $1 \mathrm{~dB}$ for 1024QAM.
\end{abstract}

\section{Introduction}

Thanks to the recent advancement of powerful forward-error correction (FEC) codes, such as low-density parity-check (LDPC) codes, the so-called turbo principle ${ }^{1-7}$ has drawn much attention to cope with various impairments in optical communications. For example, Djordjevic et al. have investigated turbo equalization to mitigate linear and nonlinear distortions ${ }^{1,2}$. In an analogous context, the second-order statistics of nonlinear distortion has been considered for sliding-window turbo equalizers ${ }^{3,4}$. Wu et al. have studied turbo carrier recovery ${ }^{5}$ with scattered pilots. Turbo differential decoding ${ }^{6}$ has been used to mitigate error propagation in differential encoding. Cycle slip issues for blind carrier/phase estimators have been dealt with by turbo slip recovery ${ }^{7}$ with hidden Markov model. In this paper, we propose another turbo receiver, referred to as turbo skew recovery, to mitigate angular skew in high-speed optical modulators. Quadrature-amplitude modulation (QAM) formats are typically generated with a triple MachZehnder structure. These modulators have inphase (I) and quadrature (Q) arms, each of which is a Mach-Zehnder interferometer. The relative phase between the I and $Q$ arms is set to $90^{\circ}$ by biasing an electro-optic phase shifter, which may be controlled with external circuitry ${ }^{8}$. Its imperfect biasing is referred to as transmitter angular skew. This skew compromises the orthogonality of the I and $Q$ components of the transmitted constellation. It should be noted that transmitter angular skew is considered as distinct from time-domain skew between the I and $Q$ arms, which may be equalized by an appropriate filter ${ }^{9,10}$. Angular skew in the receiver (where the $I$ and $Q$ arms in the optical hybrid are not at $90^{\circ}$ ) has been studied in the literature ${ }^{11}$, with the use of GramSchmidt orthogonalization providing significant benefits. Mitigation of transmitter angular skew has also been considered ${ }^{12}$ for high-order QAM. In this paper, we show a potential benefit of turbo demodulation, by comparing to those strategies.

\section{Quadrature angular skew problem}

Let $x$ be one of $M$-ary QAM constellations: e.g., $x \in\{ \pm 1 \pm \mathrm{j}\} / \sqrt{2}$ for $4 \mathrm{QAM}$, where $\mathrm{j}$ is an imaginary unit. In presence of transmitter angular skew, the transmitting constellation becomes

$$
x_{\theta}=\mathfrak{R}[x]+\sin (\theta) \mathfrak{I}[x]+\mathrm{j} \cos (\theta) \mathfrak{I}[x],
$$

where $\mathfrak{R}[\cdot], \mathfrak{I}[\cdot]$, and $\theta$ are the real-part, the imaginary-part operators, and an I-Q skew angle, respectively. Fig. 1 depicts the 1024QAM constellation for $\theta=11.45^{\circ}$. It is noted that the constellation points deviate from the ideal square-grid points according to the skew angle.

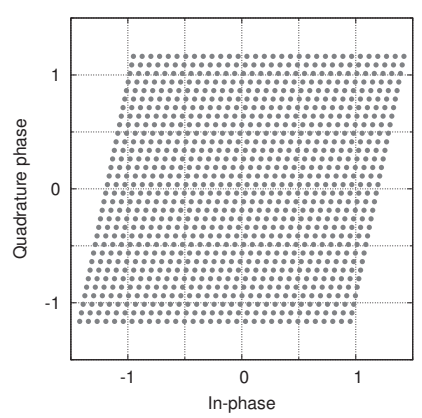

Fig. 1: 1024QAM constellation with skew angle of $\theta=11.45^{\circ}$.

After several signal processing blocks such as dispersion compensation and carrier recovery, the signal before demodulation is expressed as

$$
y=x_{\theta}+z
$$

where $z$ is an additive noise (with variance $\sigma^{2}$ ). Even without the noise source, a naïve demodulation strategy (ideal rectangular decision boundaries assuming no angular skew) suffers from a significant performance degradation in the presence of angular skew.

One demodulation strategy is the use of GramSchmidt orthogonalization process ${ }^{11}$, which makes an inverse skew for the received signal 


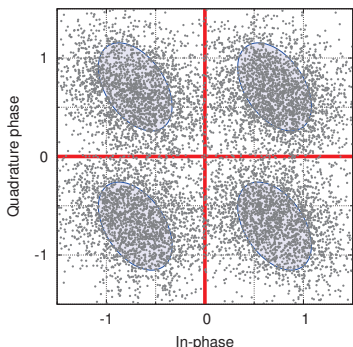

(a) Gram-Schmidt

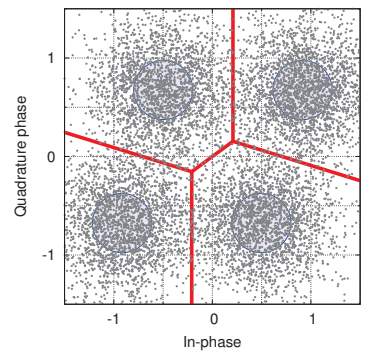

(b) K-means
Fig. 2: Demodulation strategies (4QAM with $\theta=17.2^{\circ}$ ).

with the angle of $-\theta$ as follows:

$$
y_{\theta}=\mathfrak{R}[y]+\sin (-\theta) \mathfrak{I}[y]+\mathrm{j} \cos (-\theta) \mathfrak{I}[y] .
$$

Fig. 2(a) illustrates the anti-skewed received signal constellations for $4 \mathrm{QAM}$ at a noise variance of $\sigma^{2}=0.25$. Since the mean points are recovered to a regular 4QAM, it offers a better performance than the naïve method. However, as we can see, the noise becomes non-circularly symmetric, leading to a noise enhancement.

Another strategy is to use a K-means type method $^{12}$, which determines the representative points for each cluster and data points are classified depending on which representative points are the closest. K-means method changes the decision boundary for demodulator as shown in Fig. 2(b). Because there is no noise enhancement, K-means type method offers better performance than the Gram-Schmidt method.

\section{Turbo demodulation for angle skew recovery}

We propose the use of turbo demodulation to mitigate performance degradation due to transmitter angular skew. Fig. 3 shows a schematic of the proposed turbo skew recovery, where the softdecision information is exchanged between the demodulator and LDPC decoder in a turbo loop.

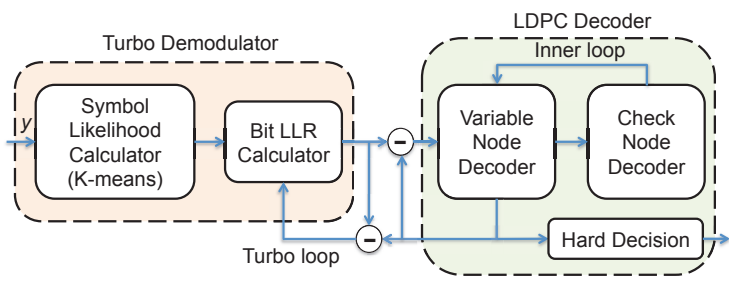

Fig. 3: Turbo skew recovery.

Provided that the additive noise follows the Gaussian distribution, the demodulator in Fig. 3 first calculates the symbol likelihood in the logarithmic domain as below (unnecessary constants discarded):

$$
d\left(x_{\theta}\right)=\frac{-1}{\sigma^{2}}\left|y-x_{\theta}\right|^{2} .
$$

This is based on the squared Euclidean distance between the received signal and the skewed QAM constellation.
The demodulator then calculates bit log-likelihood ratio (LLR) values from the distance metric and/or a priori information fed back from the LDPC decoder. The $k$-th bit LLR is calculated as

$$
L_{k}=\ln \frac{\sum_{x_{\theta}: b_{k}=1} \mathrm{e}^{d\left(x_{\theta}\right)+\frac{1}{2} \sum_{i}(-1)^{b_{i}} \lambda_{i}}}{\sum_{x_{\theta}: b_{k}=0} \mathrm{e}^{d\left(x_{\theta}\right)+\frac{1}{2} \sum_{i}(-1)^{b_{i}} \lambda_{i}},}
$$

where $\lambda_{k}$ is the soft-decision message from LDPC decoder. At the very first iteration, we have $\lambda_{k}=0$. Here, $b_{k}$ is the $k$-th bit. For numerical stability, we use the relation

$$
\ln \left(\mathrm{e}^{a}+\mathrm{e}^{b}\right)=\max (a, b)+\ln \left(1+\mathrm{e}^{-|a-b|}\right) .
$$

Given the LLR messages, the LDPC decoder employs the belief propagation between variablenode decoders (VND) and check-node decoders (CND) in an inner loop. After several LDPC decoder iterations, the extrinsic information is fed back to the demodulator to improve the LLR calculations. After a given number of outer-loop iterations, a hard decision is performed to obtain data after LDPC decoding.

\section{Results}

We used an irregular LDPC code [38400,30832] (code rate: 0.803 ), whose degree distribution is optimized to achieve $12 \mathrm{~dB}$ net coding gain by extrinsic information transfer (EXIT) chart. For all simulations, we used a total of 32 iterations for LDPC decoding. For turbo demodulation, we used 4 inner LDPC decoder iterations with 8 outer turbo iterations, resulting in a total of 32 LDPC decoder iterations.

Fig. 4 shows the post-LDPC bit-error rate (BER) performance as a function of SNR in the presence of skew angle $\theta=17.2^{\circ}$ for 4QAM. One can see that the naĩve demodulation suffers from a penalty of $0.8 \mathrm{~dB}$ at a BER of $3 \times 10^{-3}$. The Gram-Schmidt and K-means reduce the penalty to $0.4 \mathrm{~dB}$ and $0.18 \mathrm{~dB}$, respectively. Turbo demodulation further reduces the penalty to $0.08 \mathrm{~dB}$.

It is noted that the performance degradation becomes larger for higher-order QAM, due to the reduction of phase margin. Fig. 5 shows the postLDPC BER for 16QAM at a skew of $\theta=17.2^{\circ}$. Here, the curve of the naïve demodulation is not present due to a large penalty of $4.5 \mathrm{~dB}$. The penalties of Gram-Schmidt, K-means, and turbo demodulation are $0.53,0.26$, and $0.17 \mathrm{~dB}$, respectively. Those are approximately $0.08 \mathrm{~dB}$ worse than 4QAM case.

In Fig. 6, we plot the required SNR penalty as a function of skew angle $\theta$ at a post-LDPC BER of $3 \times 10^{-3}$ for 1024QAM. The naïve demodulation shows a poor tolerance to skew, while Gram-Schmidt method provides some gain, but degrades rapidly beyond $5^{\circ}$. Turbo demodulation offers the highest tolerance against the angular 


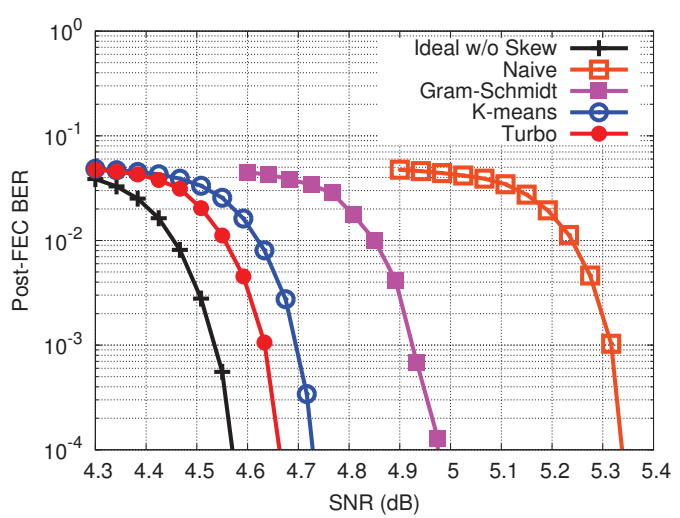

Fig. 4: Post-LDPC BER vs. SNR for 4 QAM (skew $\theta=17.2^{\circ}$ ).

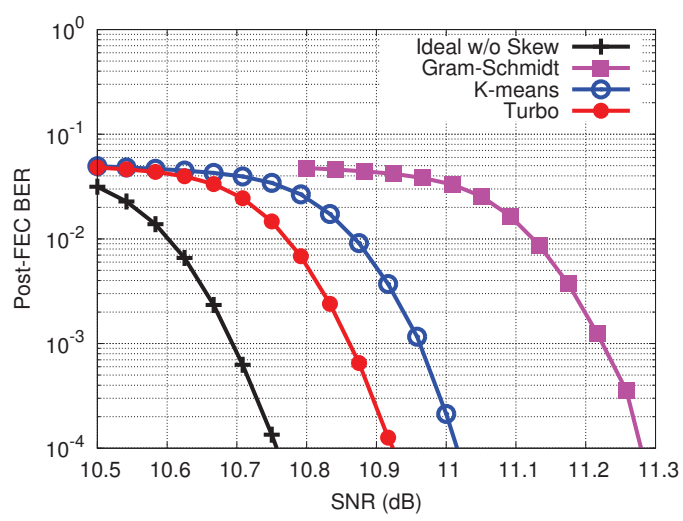

Fig. 5: Post-LDPC BER vs. SNR for 16QAM (skew $\theta=17.2^{\circ}$ ).

skew. For a skew angle of $\theta=10^{\circ}$, turbo demodulation performs better than k-means by $0.1 \mathrm{~dB}$, whereas the gain is increased to $0.3 \mathrm{~dB}$ at $\theta=25^{\circ}$. Fig. 7 shows the skew angle margin to achieve below $0.5 \mathrm{~dB}$ or $1.0 \mathrm{~dB}$ loss for required SNR as a function of modulation size from 4QAM to 1024QAM. Naïve demodulation and GramSchmidt orthogonalization are both limited strategies for lower density modulation. Turbo demodulation outperforms k-means under all cases considered here, and appears to perform better for higher-order modulation formats.

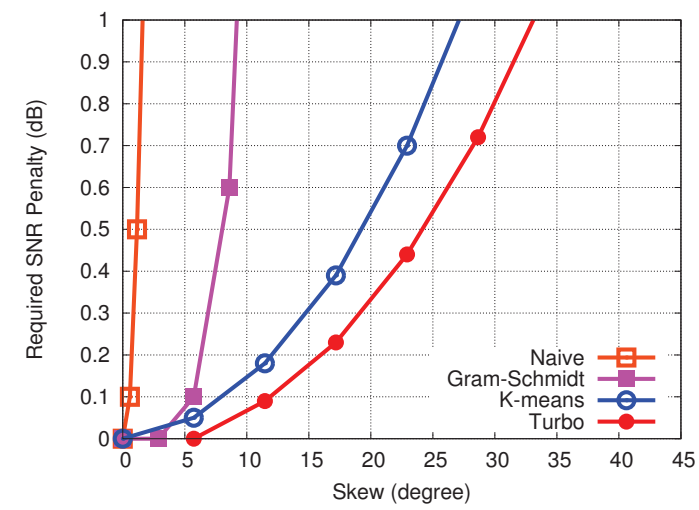

Fig. 6: Required SNR penalty vs. skew for $1024 \mathrm{QAM}$ at a BER of $3 \times 10^{-3}$.

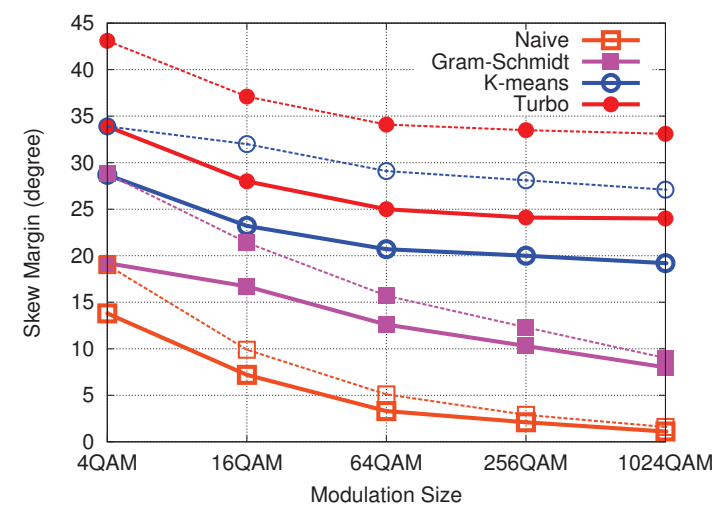

Fig. 7: Skew tolerance vs. modulation size for a BER of $3 \times 10^{-3}$ (Solid lines are results for an SNR margin of $0.5 \mathrm{~dB}$, and dashed lines are for $1 \mathrm{~dB}$ ).

\section{Conclusions}

We have investigated demodulation strategies for LDPC-coded QAM signals in the presence of transmitter angular skew. We have shown that naïve and Gram-Schmidt strategies perform poorly in particular for larger skew and higher-order modulation. K-means demodulation was found to provide a significant gain for skew beyond $10^{\circ}$. Turbo demodulation showed the best performance for all cases, with a considerable advantage over k-means demodulation.

\section{Acknowledgments}

This research is partly supported by the National Institute of Information and Communication Technology (NICT) Japan under its " $\lambda$ Reach Project".

\section{References}

[1] I. B. Djordjevic et al. "Mitigation of linear and nonlinear impairments in high-speed optical networks by using LDPC-coded turbo equalization," IEEE JSAC 26, 6 (2008) 73-83.

[2] I. B. Djordjevic et al., IEEE/OSA J. Opt. Commun. Netw., 1, 6 (2009).

[3] C. Duan et al., "A low-complexity sliding-window turbo equalizer for nonlinearity compensation," OFC, JW2A.5 (2012).

[4] T. Fujimori et al., OECC, (2013).

[5] X. Wu et al., "Iterative carrier recovery in turbo receivers with distributed pilots," CEC-Net (2011).

[6] F. Yu et al., ECOC, We.10.P1 (2011).

[7] T. Koike-Akino, K. Kojima, D. S. Millar, K. Parsons, OFC M3A.3 (2014).

[8] M. Sotoodeh, Y. Beaulieu, J. Harley, D. L. McGhan, Modulator Bias and Optical Power Control of Optical Complex E-Field Modulators IEEE JLT 29, 15 (2011) 2235-2248.

[9] M. Paskov, D. Lavery, S. J. Savory, IEEE PTL 25 24 (2013) 2446-2449.

[10] S. Randel, et al., "All-Electronic Flexibly Programmable 864-Gb/s Single-Carrier PDM-64QAM," OFC Th5C.8 (2014).

[11] I. Fatadin, S. J. Savory, D. Ives, "Compensation of Quadrature Imbalance in an Optical QPSK Coherent Receiver," IEEE PTL 20, 20 (2008) 1733-1735.

[12] S. Makovejs et al., Opt. Exp. 18, 12 (2010) 12939-12947. 